\title{
Programa de soporte a la primaria: Un análisis descriptivo y de concordancia diagnóstica entre médico/a de atención primaria y psicólogo/a.
}

Program support to primary: A descriptive analysis and diagnostic concordance between primary care physician and psychologist.

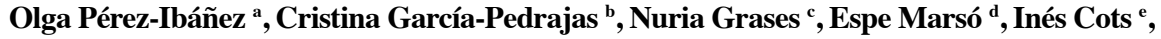
Sonia Aparicio ${ }^{\mathrm{f}}$, Susana Ochoa ${ }^{\mathrm{g}}$, Cristina Ventura ${ }^{\mathrm{h}}$.

${ }^{a}$ Centro de Salud Mental Castelldefels, Parc Sanitari San Joan de Déu, Barcelona, España. ${ }^{b}$ Residente de Psicología Clínica, Centro de Salud Mental de Cornellà, Parc Sanitari San Joan de Déu, Barcelona, España. ${ }^{c}$ Residente de Psicología Clínica, Centro de Salud Mental Cerdanyola, Parc Sanintari San Joan de Déu, Barcelona, España. ${ }^{d}$ Centro de Salud Mental Ripollet, Cerdanyola y Montcada i Reixach, Parc Sanitari San Joan de Déu, Barcelona, España. ${ }^{e}$ Centro de Salud Mental El Prat de Llobregat, Parc Sanitari San Joan de Déu, Barcelona, España. ${ }^{f}$ Centro de Salud Mental Garraf, Parc Sanitari San Joan de Déu, Barcelona, España. ${ }^{g}$ Coordinadora de la Unitat de Recerca i Desenvolupament, Parc Sanitari Sant Joan de Déu, Barcelona, España. ${ }^{h}$ Centro de Salud Mental Cornellà, Parc Sanitari San Joan de Déu, Barcelona, España.
\end{abstract}

Correspondencia: O.Pérez Ibáñez(operez@pssjd.org)

Recibido: 24/02/2014; aceptado con modificaciones: 23/07/2014

RESUMEN: Objetivos: Describir las características del Programa de Soporte a la Primaria (PSP) y analizar la concordancia diagnóstica entre Médicos de Atención Primaria (MAP) y Psicólogos/as del programa. Metodología: Estudio descriptivo longitudinal realizado en 2010 en 7 CAPs de Barcelona. La muestra son 769 pacientes derivados a psicología. Se utilizan estadísticos descriptivos para analizar las variables sociodemográficas y la kappa de Cohen para valorar la concordancia.

Resultados: El tiempo de espera medio entre derivación y primera visita es 28 días. La media de intervenciones por paciente es 3 visitas. Un $27 \%$ son dados de alta, un 20\% abandonan. Existe una baja concordancia global entre MAP y Psicólogo/a.El diagnóstico más frecuentemente realizado por los MAP es Trastorno de Ansiedad.Los psicólogos/as diagnostican con igual frecuencia Trastornos Ansiosos (21\%), Depresión (20.2\%) y Trastornos Adaptativos (19.6\%).

Conclusiones: El PSP ofrece intervenciones breves y especializadas desde Atención Primaria.La baja concordancia indica la necesidad de aumentar espacios de interconsulta.

PALABRAS CLAVE (DECS): Salud Mental; Atención Primaria; Psicólogo; Concordancia diagnóstica; Médico de Atención Primaria.
ABSTRACT: Background: The objective is to describe characteristics of Program Support to Primary(PSP) and analyze diagnostic concordance between Primary Care Physicians(PCP) and psychologists.

Method: A descriptive, longitudinal study conducted in 2010 in 7 Barcelona's primary care centers. The sample of 769 patients referred to psychology(referral form issued by doctors). Descriptive statistics, Cohen's kappa and percentage agreement were used to analyze demographic variables, to assess agreement. Results: The average waiting time between referral and first visit is 28 days. Mean interventions per patient is 3 visits. $27 \%$ of patients are discharged, $20 \%$ drop. It is a low overall concordance between PCP and psychologist, the most common diagnosis made by the PCP is anxiety disorder.Psychologists diagnosed with the same frequency anxiety disorders $(21 \%)$, depression $(20.2 \%)$ and adaptive disorders $(19.6 \%)$.

Conclusions: PSP offers brief and specialized interventions in Primary Care.Low concordance indicates a need to increase opportunities for communication between professionals through tools such as consultation.

KEY WORDS (MESH): Mental Health; Primary Care; Psychologist; Diagnostic concordance; Physician of Primary Care. 
ORIGINALES Y REVISIONES

\section{Introducción}

La Atención Primaria de Salud (AP) es la puerta de entrada de la población al Sistema Sanitario de Salud. Es el recurso al que acceden en primera instancia, no solo la mayoría de personas con patología somática, sino también aquéllas con problemas de salud mental y/o psicosociales. Constituye el acceso al sistema de salud mental para el $70 \%$ de la población (1)

La gran prevalencia de trastornos de salud mental no graves, las adicciones y los denominados "problemas de la vida", generan una alta demanda de atención a los Equipos de Atención Primaria (EAP) (2-4) . En Europa, la prevalencia de los trastornos mentales en este contexto ha estado estimada entre el 20-55\% (5).

A nivel internacional, existe un reconocimiento claro por parte de numerosos expertos/as y sociedades científicas acerca de la necesidad de incorporar profesionales de la Salud Mental a los equipos de Atención Primaria (6-7). Tal y como cita Bower (8), uno de los modelos más estudiados y con mayor respaldo científico es el de la asistencia integrada, el modelo "collaborative care", que se basa en la asistencia especializada y atención formativa al equipo de Atención Primaria.

En este sentido, a nivel nacional se han realizado varios estudios que evalúan la coordinación entre la Atención Primaria y la especializada, evaluando variables como son las características de la derivación (motivo de consulta, datos sociodemográficos), tiempo de espera y concordancia diagnóstica entre profesionales de primaria y especialistas (9). En estos estudios, como los realizados por Miranda-Chueca (10) o Martín-Jurado y col. (11), el contexto desde donde intervienen los especialistas son los servicios de salud mental, ya que en la gran mayoría de estos estudios los Centros de Atención Primaria (CAP) no cuentan con el Programa de Soporte a la Primaria (9-13). Sánchez González y col. (14) también encuentran una baja concordancia entre el médico/a y los/ as psicólogos/as del CSMA. La diferencia en nuestro estudio es que hemos evaluado la concordancia entre el psicólogo /a y el médico/a, ambos en el mismo contexto físico: el CAP.

En el año 2006 en Catalunya se crea el Programa de Soporte a la Primaria (PSP), dentro del Plan Director de Salud Mental y Adicciones. Éste propone un modelo de colaboración y apoyo entre el CAP y el Centro de Salud Mental de Adultos (CSMA) con el objetivo de mejorar la atención a las necesidades psicológicas de los usuarios/as. Esta labor se lleva a cabo a través de un equipo de profesionales especialistas, formado por un/una psiquiatra, psicólogo/a, y enfermero/a que se desplazan al CAP. El programa tiene una doble vertiente: formativa-apoyo técnico y terapéuticaasistencial. La intervención formativa se concreta en sesiones clínicas y espacios de interconsulta (asesoramiento al profesional). La intervención terapéutica-asistencial comprende desde visitas conjuntas con los profesionales de AP hasta intervenciones psicoterapéuticas breves. 
El programa permite la detección y derivación de patologías que requieren un abordaje especializado desde el CSMA, potencia los recursos de AP para la atención psicológica de complejidad leve-moderada y ofrece soporte asistencial a los CAP con intervenciones psicofarmacológicas y terapias psicológicas breves.

El presente artículo tiene como objetivo analizar las intervenciones que realiza el/la psicólogo/a dentro de este programa, excluyendo la intervención de Psiquiatría y Enfermería en Salud Mental. Se exponen las características sociodemográficas y clínicas de los pacientes atendidos por psicólogo/a en 7 CAPs de la provincia de Barcelona a lo largo del año 2010. También se analiza el índice de concordancia diagnóstica entre los MAP y los/las psicólogos/as del programa. Desde la implementación de este programa no se han descrito estudios que evaluaran el impacto de éste. Este proyecto pretende conocer cuáles son las características de la intervención del psicólogo en el programa y tener una base que permita planificar intervenciones futuras dirigidas a su mejora.

\section{Material y método}

Se trata de un Estudio Descriptivo Prospectivo realizado a lo largo del año 2010 en siete Centros de Atención Primaria (CAP) de la provincia de Barcelona.

Aquellos/as pacientes derivados al psicólogo/a del programa PSP a través de la hoja de derivación emitida por los MAPS $(N=769)$ fueron seguidos durante un año o final del proceso con la intención de recoger información sobre la intervención.

Se excluyeron del estudio aquellos/as pacientes derivados exclusivamente del MAP a otro profesional especializado no psicólogo/a (psiquiatría PSP, enfermería PSP) o cualquier profesional del Centro de Salud Mental Adultos.

Para la recogida de datos basal se utilizó la información de la hoja de derivación (donde se reflejan el motivo de consulta y el diagnóstico CIE 10 de derivación a criterio del MAP). En la primera visita se recogieron una serie de datos por parte del psicólogo/a, incluyendo la revisión del diagnóstico realizado por el MAP. Todos/ as los/as pacientes fueron seguidos durante su vinculación al PSP y en las sucesivas visitas se siguió cumplimentado la información del estudio (ver figura 1).

En el protocolo de evaluación se recogió la siguiente información. En el momento basal:

- Características socio-demográficas de la muestra (edad, sexo, situación laboral, antecedentes psicopatológicos y motivo por el que se consulta).

- Motivo de consulta por parte del MAP. Esta variable la clasificamos en tres grupos: por clínica psicopatológica, por problemas familiares, laborales y/o sociales y finalmente por otros motivos (tribunal médico, informes, etc.) 
- Fuente de derivación: Médico de cabecera o derivación a través de otro profesional del equipo PSP (enfermero o psiquiatra)

- Diagnóstico de derivación del MAP basados en criterios CIE-10. Se utiliza el diagnóstico principal en el caso de comorbilidad.

- Diagnóstico CIE 10 por parte del psicólogo/a del PSP

Figura 1:

Secuencia para la recogida de datos del perfil de pacientes atendidos

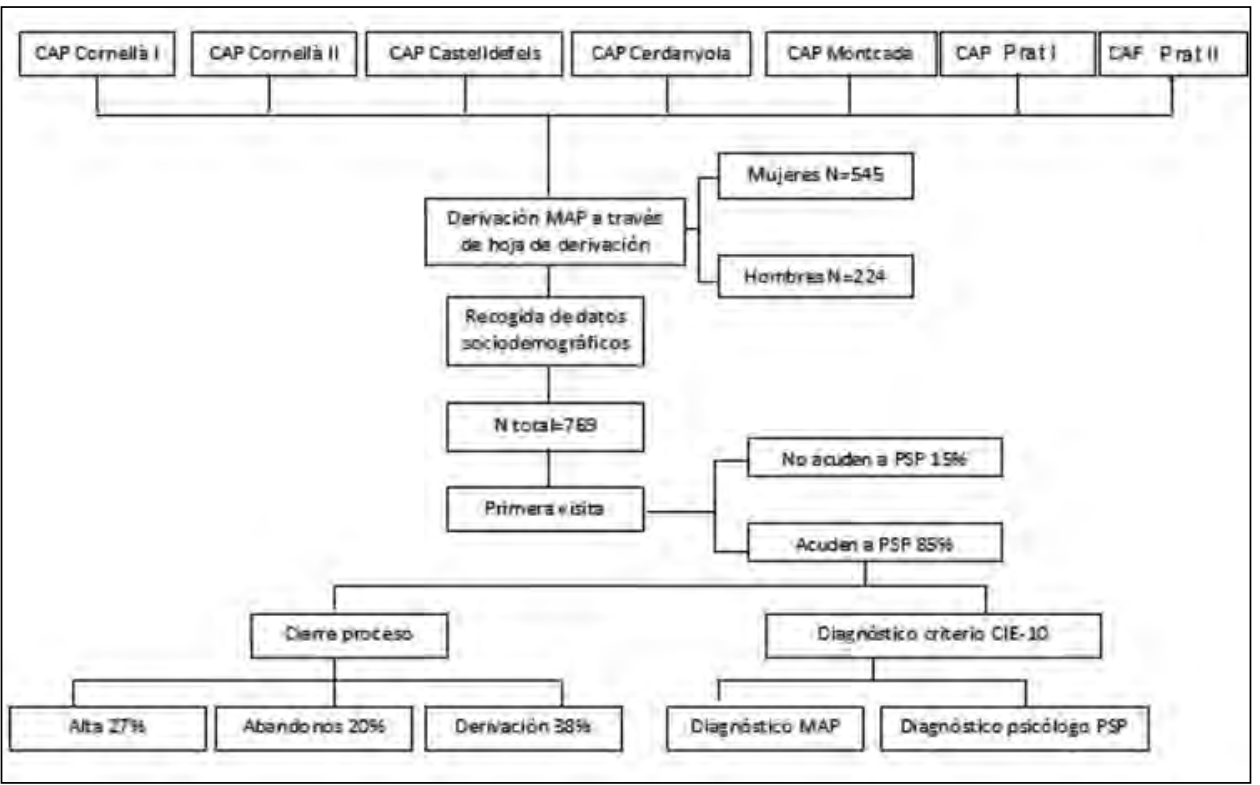

En las siguientes visitas se recogió información sobre:

- Número total de visitas de la intervención psicológica (primera visita más visitas sucesivas)

- Tipos de visitas (individual, familiar, mixta, interconsulta)

- Cierre de proceso (no presentado primera visita, por alta, abandono o derivación a otro recurso)

El análisis estadístico se llevó a cabo a través de estadística descriptiva. Se calculó la kappa de Cohen y el Porcentaje de Acuerdo para valorar el acuerdo entre los diagnósticos de los profesionales de Atención Primaria y de Salud Mental.

El proyecto de investigación fue aprobado por el comité de investigación y de ética del Parc Sanitari Sant Joan de Déu (PSSJD). A todas las personas participantes se solicitó el consentimiento informado. 
ORIGINALES Y REVISIONES

\section{Resultados}

La muestra la componen 769 pacientes, de los cuales 224 son varones (29\%) y 545 son mujeres (71\%), todos ellos mayores de 18 años. La media de edad es de 41 años $(\mathrm{DE}=13,46)$.

En la Tabla 1 se presentan las características sociodemográficas (edad, sexo, situación laboral) de la muestra. Respecto a la situación laboral resaltar que un 30\% están en situación de Incapacidad Laboral Transitoria (ILT). De éstos un 38,1\% cumplen criterios de Trastorno Mental Leve/Moderado (CIE-10) y un 61,9\% presentan síntomas no clasificables dentro de ningún trastorno mental. En la misma tabla, se incluyen las variables clínicas, como son antecedentes psicopatológicos del usuario previos a la consulta y los tipos de motivo de consulta.

Tabla 1:

Características sociodemográficas y clínicas de la muestra

\begin{tabular}{|llcc|}
\hline \multirow{2}{*}{ Edad } & & Media & Desviación típica \\
& & 41 & 13,46 \\
\multirow{4}{*}{ Género } & Varón & N & \% \\
& Mujer & 224 & 29 \\
\multirow{4}{*}{ Situación laboral } & ILT* & 545 & 71 \\
& No ILT & 222 & 30 \\
Antecedentes & Tratado por MAP & 508 & 70 \\
psicopatológicos & Tratado por otros** & 195 & 29 \\
personales & Sin antecedentes & 226 & 34 \\
\multirow{2}{*}{ Motivo consulta } & No explorados*** & 247 & 37 \\
& Psicopatológicos & 110 & 13,2 \\
& Problemas familiares & 452 & 59 \\
& Otros**** & 267 & 35 \\
\hline
\end{tabular}

*ILT: Incapacidad Laboral Transitoria

**Tratado por otros profesionales sanitarios

***Casos no explorados al pertenecer a los casos que no acuden a primera visita

****Otros: problemas laborales, sociales, demandas de informes o duelos.

En la Tabla 2 se detallan las características del trabajo del psicólogo/a dentro del programa, valorando el número de visitas por intervención, el tipo de visitas y el cierre del proceso. La media del número de visitas ha sido de 2,53. El tipo de visita más frecuente ha sido la visita individual (70\%), seguida de las interconsultas (24\%). Respecto al cierre de proceso, un $38 \%$ de pacientes fueron derivados a otros dispositivos, mientras que el $27 \%$ de pacientes fueron dados de alta del programa. El tiempo de espera medio entre la derivación y la primera visita es de 28,97 días. 
Tabla 2:

Tipo de atención por parte del psicólogo del PSP

\begin{tabular}{|llcc|}
\hline \multirow{2}{*}{ Número de visitas } & Media & Desviación típica \\
& & 2.53 & 2.04 \\
\multirow{4}{*}{ Tipo de visita } & $\mathbf{N}$ & $\mathbf{\%}$ \\
& & 462 & 70 \\
& individual & 32 & 5 \\
& familiar & 6 & 1 \\
Cierre & mixta & 160 & 24 \\
& interconsulta & 196 & 27 \\
& alta & 110 & 15 \\
& no presentado a $1^{\text {a }}$ visita & 140 & 20 \\
& abandono & 277 & 38 \\
\hline
\end{tabular}

En la Tabla 3 se especifican los diagnósticos principales que fueron valorados tanto por el/la MAP (previo a la derivación al PSP) como del psicólogo/a una vez que el/la paciente se incluye dentro del programa. Además se indican los índices de correlación entre los diagnósticos valorados por ambos profesionales. Los diagnósticos más frecuentes realizados por los/as MAP son: Trastorno de Ansiedad (28,4\%), sin diagnóstico (18,6\%) y Trastorno depresivo (14,6\%), y los diagnósticos más frecuentes realizados por los psicólogos/as fueron: Trastorno Ansiedad (21,4\%), Trastorno Depresivo (20,2\%), y Trastorno Adaptativo (19,6\%). Para analizar la correlación se incluye el índice de kappa y el índice de acuerdo. El acuerdo entre el/la MAP y el psicólogo/a fue bajo en todos los diagnósticos, especialmente en los trastornos de personalidad, trastorno por dependencia de tóxicos y trastorno psicótico, en los que el kappa de acuerdo es inferior a 0,1 .

Tabla 3:

Diagnósticos según MAP y psicólogos/as

\begin{tabular}{|lcrrrrrr|}
\hline & Diagnostico MAP & \multicolumn{2}{c}{ Diagnóstico PSP } & \multicolumn{2}{c|}{ \% de acuerdo } & Kappa \\
\hline & $\boldsymbol{N}$ & $\mathbf{\%}$ & $\boldsymbol{N}$ & $\mathbf{\%}$ & $\boldsymbol{N}$ & \multicolumn{1}{c|}{$\%$} & \\
\hline Depresión & 112 & 14,6 & 103 & 20,2 & 43 & 41,7 &, 303 \\
\hline Tóxicos & 5 &, 7 & 11 & 2,2 & 0 & 0 & 0 \\
\hline Psicosis & 13 & 1,7 & 2 &, 4 & 0 & 0 & 0 \\
\hline T.Ansiedad & 217 & 28,4 & 109 & 21,4 & 68 & 62,4 &, 282 \\
\hline T.Mixto & 69 & 9,0 & 32 & 6,3 & 13 & 40,6 &, 213 \\
\hline T.Adaptativo & 53 & 6,9 & 100 & 19,6 & 19 & 19 &, 174 \\
\hline T.Alimentación & 14 & 1,8 & 7 & 1,4 & 2 & 28,6 &, 181 \\
\hline T.Personalidad & 13 & 1,7 & 29 & 5,7 & 2 & 6,9 &, 074 \\
\hline T.Somatomorfo & 42 & 5,5 & 4 &, 8 & 4 & 100 &, 166 \\
\hline Probl.familiares & 76 & 9,9 & 91 & 7,8 & 31 & 34,1 &, 295 \\
\hline Probl.laborales & 9 & 1,2 & 14 & 2,7 & 4 & 28,6 &, 338 \\
\hline Sin diagnóstico & 142 & 18,6 & 8 & 1,6 & 3 & 37,5 &, 021 \\
\hline
\end{tabular}


ORIGINALES Y REVISIONES

\section{Discusión}

En el presente trabajo hemos analizado tanto las características descriptivas de la muestra, como la concordancia diagnóstica entre los profesionales implicados en el PSP. Así, encontramos una baja concordancia global entre los diagnósticos del MAP y del psicólogo/a en el programa. Estos resultados coinciden con otros estudios, sin embargo la diferencia es que en este caso el psicólogo/a está ubicado en el CAP, mientras que en el resto de estudios el psicólogo/a está ubicado en el CSMA (15-19).

La diferencia de correlación es esperable ya que se trata de dos equipamientos profesionales distintos, con formación, procedimientos y fines de valoración diferenciados: El/la MAP diagnostica para derivar correctamente, y el psicólogo/a diagnostica para intervenir adecuadamente. Por ello partimos del principio de que alcanzar una mayor concordancia contribuye a una mejor derivación, y por tanto a un trabajo más eficaz y efectivo.

El principal motivo de consulta, son los problemas psicopatológicos, lo que nos hace pensar que la derivación ha sido adecuada dado que el objetivo del programa es atender Trastorno Mental Leve/Moderado. Cuando nos referimos a este tipo de problemas aludimos a aquellos síntomas clasificables dentro de los criterios diagnósticos de la CIE- 10 (Clasificación Internacional Europea). La bibliografía revisada constata que los trastornos emocionales son el motivo de consulta más habitual en AP (1, 3-4, 20-21). Estos resultados concuerdan con los del presente estudio, donde el trastorno más prevalente es el de ansiedad y seguidamente el trastorno depresivo.

Cuando nos referimos a problemas familiares u otros (laborales, sociales, demandas de informes...), aludimos a problemas de la vida cotidiana que están contemplados en la codificación adicional CIE-10 códigos Z. Esto forma parte del trabajo que empieza abordando el/la MAP y que requiere de consulta puntual por parte del psicólogo/a especialista. El intervenir en estas dificultades emociones asociadas a factores socio-familiares forma parte de la labor preventiva del psicólogo/a, evitando que las dificultades se transformen en trastornos.

El diagnóstico más frecuente en los médicos/as ha sido el de trastorno de ansiedad. Sin embargo, los psicólogos/as diagnostican con mayor frecuencia el trastorno de ansiedad, junto con el trastorno depresivo y los trastornos adaptativos. Se observa que en muchos de estos casos orientados como Trastorno de Ansiedad o Trastorno Depresivo por el médico/a, acaban siendo diagnosticados por el psicólogo/a como Trastorno Adaptativo, coincidiendo con otros autores (15). Esto podría entenderse desde la consulta médica, que ha de centrarse en el síntoma, a diferencia de la consulta psicológica que hace una evaluación psicodiagnóstica.

La categoría registrada de "sin diagnóstico definitivo" puede significar que el MAP tenga dudas para poder concretar un diagnóstico psicopatológico; hay que tener 
ORIGINALES Y REVISIONES

en cuenta la presión asistencial a la que se ve sometido el/la MAP que dificulta la tarea. También destaca la mayor dificultad para diagnosticar trastornos de personalidad, dado que entendemos que se trata de un diagnóstico más específico que corresponde al profesional de Salud Mental.

El 37\% de la muestra no había consultado previamente en ningún dispositivo por sintomatología psicopatológica. Este dato es importante ya que indica que un tercio de la población que atendemos debuta por primera vez con dicha clínica, con lo cual las intervenciones de los psicólogos/as en este momento pueden ser decisivas en para evitar la cronicidad (3).

A nivel descriptivo se encuentran similitudes con respecto a la bibliografía publicada $(4,6,8)$. Así, se observa una mayor prevalencia de mujeres que consultan en Atención Primaria (22), lo cual también se objetiva en las derivaciones realizadas al psicólogo/a. La media de intervenciones para cada paciente que es de tres, se explica debido a que la intervención es prioritariamente focalizada, breve, y centrada en la demanda: Desde la primera visita el psicólogo/a está realizando intervención psicoterapéutica. Otro aspecto importante es la función de cribaje que desde el programa realiza el psicólogo/a para discriminar la patología leve/moderada de la grave, dado que esta última debe ser atendida desde un CSMA. Además, a través de la interconsulta, el psicólogo/a orienta hacia otros recursos de la comunidad. Esto nos explica el número de intervenciones breves y concisas que realiza el psicólogo/a en los dos niveles: interconsulta y asistencia directa. Hay que tener en cuenta que en ocasiones la primera visita ya consta como alta al no detectarse clínica psicopatológica que justifique intervención. Este punto nos hace plantear la importancia de mejorar la vertiente formativa y de comunicación entre profesionales en cuanto al análisis de las derivaciones a través de la interconsulta. Por otro lado, el propio rol del psicólogo/a como especialista permite en muchos casos encuadrar el motivo de demanda del paciente en un contexto de normalización y contención que a veces desencalla y resuelve el cuadro en una única visita sin necesidad de seguimiento.

El porcentaje de absentismo (pacientes no presentados a primera visita) sugiere la importancia de reformular cuál ha sido el criterio de derivación del MAP y sus expectativas así como profundizar en las motivaciones del paciente. Por nuestra experiencia clínica la impresión es que podría estarse dando el efecto de derivaciones motivadas por el médico/a más que por el propio/a paciente. El poder optimizar los espacios de interconsulta podría mejorar la adecuación de la derivación y su intervención en el programa. Otra hipótesis podría ser que durante el tiempo de espera previo a la primera visita, el paciente resolviera con sus propios recursos la conflictiva por la cual consultaba. Aunque el tiempo de espera entre la derivación del médico y la primera visita con psicología es de menos de un mes, el tratamiento realizado a partir de la primera visita suele ser intensivo ofreciendo un tiempo entre visitas más reducido. Durante el proceso de tratamiento algo menos de una cuarta parte de los pacientes 
abandonan, esto podría deberse entre otros motivos, a que el paciente atendido en un contexto médico/a, es menos grave y puede tener la idea de una consulta puntual y aislada de remisión sintomática.

El impacto de este trabajo conjunto en las ABS es un trabajo pendiente de operativizar, si bien la impresión subjetiva por parte de los psicólogos/as que participamos en el programa es de una satisfactoria aceptación y adaptación. Los/as MAP se muestran cada vez más receptivos a participar en los espacios de interconsulta de reunión general, así como a propiciar espacios de interconsulta de casos individuales, se implican más en los casos en los que hay impacto emocional con o por la enfermedad que les traen, lo que hace que soliciten más formación sobre manejo de entrevista para afinar más en la derivación a la especializada. Por otro lado, la impresión sobre la respuesta del paciente es la de la vivencia de recepción de una atención integral y global, abordándose las distintas dimensiones que como persona trae a consulta como fruto de su malestar, físico y/o psíquico.

El objetivo del PSP ha sido desde su implementación, ofrecer una cartera de servicios compartida entre AP y SM propiciando un mayor diálogo interno entre profesionales para mejorar la concordancia (23), reformular criterios de derivación a través de la interconsulta y optimizar la exploración sintomática por parte del MAP. Integrar la figura del psicólogo/a especialista en el CAP para analizar la demanda de problemas emocionales, de la vida y/o problemas de salud mental está siendo una experiencia enriquecedora. El poder compartir, sirve de ayuda y soporte mutuo en la tarea de reflexión sobre qué le sucede al paciente, y aportar herramientas que permitan la gestión y el manejo del malestar de la vida de los pacientes.

\section{BIBLIOGRAFÍA}

(1) Pastor, J. El psicólogo en AP: un debate necesario en el sistema nacional de salud. Papeles del psicólogo 2008; 29 (3): 271-290.

(2) Generalitat de Catalunya. Departament de Salut. Salut mental i qualitat de vida. Pla Director i Addiccions. Departament de Salut 2006.

(3) Ortiz-Lobo A, González-González R, Rodríguez-Salvanés F. La derivación a Salud Mental de pacientes sin un trastorno psíquico diagnosticable. Atención Primaria 2006; 38 (10): 563-569.

(4) Martínez M, Dolz M, Alonso J, Luque I, Palacín C, Bernal M, et al. Prevalencia de los trastornos mentales y factores asociados: resultados del estudio ESEMeD-España. Medicina Clínica 2006; 12 (126): $445-451$.

(5) Serrano-Blanco A, Palao DJ, Luciano JV, Pinto-Meza A, Luján L, Fernández A, et-al. Prevalence of mental disorders in primary care: results from the diagnosis and treatment of mental disorders in primary care study (DASMAP). Soc Psychiatry Psychiatr Epidemiol 2010; 45: 201-10.

(6) Thielke S. Health psychology in primary care: recent research and future directions. Psychology Research and Behavior Management 2011; 4: 59-68. 
ORIGINALES Y REVISIONES

(7) Tanielian TL, Pincus HA, Dietrich AJ, Williams JW Jr, Oxman TE, Nutting P, et al. Referrals to psychiatrists. Assessing the communication interface between psychiatry and primary care. Psychosomatics 2000; 41: 245-52.

(8) Bower, P. Primary care mental health workers: models of working and evidence of effectiveness. British Journal of General Practice 2002; 52: 926-933.

(9) Espinosa-Sabina L, Castilla-Pérez MP. Estudio descriptivo de las derivaciones urgentes a una unidad de salud mental. Rev. Asoc. Esp. Neuropsiq 2002; 22 (83): 111-123.

(10) Miranda-Chueca I, Peñarrubia-María MT, García-Bayo I, Caramés-Durán E, Soler-Vila M, Serrano-Blanco A. ¿Cómo derivamos a salud mental desde AP? Atención Primaria. 2003; 32 (9): 524-530.

(11) Martín-Jurado A, de la Gándara Martín JJ, Castro Carbajo S, Moreira Hernández A, Sánchez-Hernández J. Análisis de concordancia de las derivaciones de Atención Primaria a Salud Mental. Semergen 2012.

(12) Franquelo P, García D, Moya P, Lema J. Tasa de derivación y factores asociados a la calidad de la hoja de derivación en Atención Primaria. Rev Clin Med Fam 2008; 2 (5): 199-205.

(13) Goñi A, García E, Landa N, Lizasoain E. Análisis de las derivaciones desde atención primaria a salud mental. Anales de Psiquiatría 2008; 24(2)

(14) Sánchez-González D, Vega-Moreno D, Soto-Lumbreras A, Andreu i Gràcia A, CastellsRovira M, Navarro-Martín D, et al. ¿En qué ocasiones el médico de familia solicita un tratamiento psicológico para sus pacientes?: análisis descriptivo y de concordancia entre diagnósticos. C. Med. Psicosom 2011; 97: 31-37.

(15) Landa N, Goñi A, García de Jalón E, Lizasoain E. Concordancia en el diagnóstico entre atención primaria y salud mental. Atención Primaria. 2008; 40 (6): 285-289.

(16) Cano-Vindel. Intervención psicológica en atención primaria: una experiencia piloto. Infocop 2013; 60: 7-9

(17) Marsó E, Ventura C, García-Pedrajas C, Pérez, O. y Grases, N. (2012, Febrero). ¿Hay concordancia entre los diagnósticos de los médicos/as de AP y los psicólogos/as?. Paper presented at Mental Health and Family Medicine working together, Granada, España

(18) Ordoñez MP, Gómez-Ullate C. Estudio de la demanda a nuestra Unidad de salud Mental: la calidad de los partes de interconsulta y la concordancia. Anales de psiquiatría 2009; 25(2): 70-78.

(19) Rodríguez-Caravaca G, Villar del Campo I. Concordancia diagnóstica entre atención primaria y atención especializada tras consulta urgente. Atención Primaria 2000; 25(5): 292-296.

(20) Ventura C, Pérez O, Marso E, Grases N, Cots, I, Ochoa, S. y García-Pedrajas C. Diferencias de género entre las personas atendidas en el PSP en el año 2010. Póster presentado en III Congreso Català de la Dona i Salut Mental, Barcelona, España. Octubre, 2012.

(21) Grases N, Pérez O, Marso E, Ventura C, Cots I, Ochoa S, García-Pedrajas C. Concordancia en el diagnóstico en función del género de los médicos de Atención Primaria. Póster presentado en III Congreso Català de la Dona i Salut Mental, Barcelona, España. Octubre, 2012.

(22) Rodríguez-Muñoz C, Cebrià-Andreu J, Corbella-Santomà S, Segura-Bernal J, Sobreques-Soriano J. Rasgos de personalidad y malestar psíquico asociados a los pacientes hiperfrecuentadores de consultas de Atención Primaria. Atención Primaria, 2003; 13: 143-150.

(23) Cuesta L, Alberto M, Prat J, Simón O, Marcos R. Adecuación del diagnóstico, tratamiento y derivación de la patología psiquiátrica en asistencia primaria. Utilidad y/o necesidad de coordinación. En: V Congreso Nacional de Psiquiatría; Zaragoza 18-21 de octubre 2000. 